\title{
Efficacy of self-heating calf sleeves for preventing deep vein thrombosis in lung cancer patients who undergo video-assisted thoracoscopic surgery lobectomy
}

\author{
Shenghua Chen", Wenwen Xu", Saiqi Song, Xiaojun Wang \\ Department of Operating Room, Shanghai Pulmonary Hospital, Tongji University School of Medicine, Shanghai, China \\ Contributions: (I) Conception and design: X Wang, S Song; (II) Administrative support: X Wang, S Song; (III) Provision of study materials or patients: \\ All authors; (IV) Collection and assembly of data: All authors; (V) Data analysis and interpretation: All authors; (VI) Manuscript writing: All authors; \\ (VII) Final approval of manuscript: All authors. \\ "These authors contributed equally to this work. \\ Correspondence to: Xiaojun Wang; Saiqi Song. Department of Operating Room, Shanghai Pulmonary Hospital, Tongji University School of Medicine, \\ Shanghai 200433, China. Email: woailuyue0701@126.com; zhaoyunfeng518@126.com.
}

Background: Deep vein thrombosis (DVT) poses a considerable perioperative risk in patients who undergo surgical procedures. This study set out to determine the efficacy of self-heating calf sleeves (SHCSs) in preventing postoperative DVT in lung cancer patients undergoing VATS lobectomy.

Methods: This study included 557 participants who underwent video-assisted thoracoscopic surgery (VATS) lobectomy between June, 2018 and June, 2019. The participants were randomly divided into two groups: the SHCS group and the control group. SHCS was applied to the patients intraoperatively. Each patient was subjected to Doppler ultrasound and D-dimer examination preoperatively and postoperatively. Signs and symptoms of DVT were monitored daily. Incidences of DVT were compared between the two groups.

Results: Based on Color Doppler ultrasonography performed on day 2 after surgery, 42 of the 276 patients (15.20\%) in the control group developed DVT, compared to 12 out of 281 patients (4.27\%) in the SHCS group. There was significant difference in the incidence of DVT between the two groups $(\mathrm{P}<0.0001)$. The use of SHCSs significantly enhanced venous blood flow compared to when no mechanical prophylaxis was used $(\mathrm{P}<0.001)$. No significant difference in plasma $\mathrm{D}$-dimer level was observed between the two groups. No SHCS-related complications occurred in the study.

Conclusions: This study demonstrate that SHCSs are a satisfactory form of DVT prophylaxis method that can improve venous blood flow and thus alleviate venous stasis in the lower extremities. SHCSs offer a simple, inexpensive, and generally complication-free prophylactic method for lung cancer patients undergoing VATS lobectomy.

Trial registration: ChiCTR1900022043.

Keywords: Deep vein thrombosis (DVT); self-heating calf sleeve (SHCS); lung cancer; video-assisted thoracoscopic surgery (VATS)

Submitted Mar 27, 2020. Accepted for publication Jul 22, 2020.

doi: 10.21037/apm-20-1165

View this article at: http://dx.doi.org/10.21037/apm-20-1165 


\section{Introduction}

Deep vein thrombosis (DVT), which refers to the formation of a thrombus formed in a deep vein, typically in the lower limbs or pelvis, is a life threatening event $(1,2)$. Without timely intervention, DVT ultimately leads to pulmonary embolism (PE) and has a mortality rate as high as 30\% (3). Strong risk factors for venous thromboembolism include active cancer, major surgery, reduced mobility, older age, and a history of DVT (4). Among lung cancer patients, those who undergo surgery are at increased risk of developing DVT (5-8); as many as $16.2 \%$ of these patients develop DVT (9).

The diagnosis of DVT is usually based on estimation of clinical probability, the level of plasma D-dimer, and Doppler ultrasonography. Due to the difference in symptoms between patients and the unreliability and low sensitivity of laboratory testing, diagnosing DVT presents a clinical challenge, and routine thromboprophylaxis is fundamental to reducing the perioperative mortality (10). Mechanical methods of DVT prophylaxis, such as intermittent pneumatic compression (IPC) and graduated compression stockings (GCSs), have been widely used perioperatively, as they are free of the hemorrhagic complications associated with pharmacologic prophylaxis. However, issues with patient compliance often arise when these methods are used.

To address the issue of compliance associated with the existed mechanical DVT prophylaxis methods, we invented a simple and economic mechanical DVT prophylaxis device, a self-heating calf sleeve (SHCS), which could maintain an even temperature of about $38^{\circ} \mathrm{C}$ to prevent the stasis of the blood flow. This study aimed to evaluate the efficacy and the potential application of our SHCS in the prevention of DVT in lung cancer patients who underwent lung resection by video-assisted thoracoscopic surgery (VATS).

We present the following article in accordance with the CONSORT reporting checklist (available at http://dx.doi. org/10.21037/apm-20-1165).

\section{Methods}

\section{Patient recruitment}

A total of 557 consecutive lung cancer patients (age range, 35-81 years old) who underwent VATS lobectomy in Shanghai Pulmonary Hospital between June, 2018 and June, 2019 were included in this study. A flow diagram

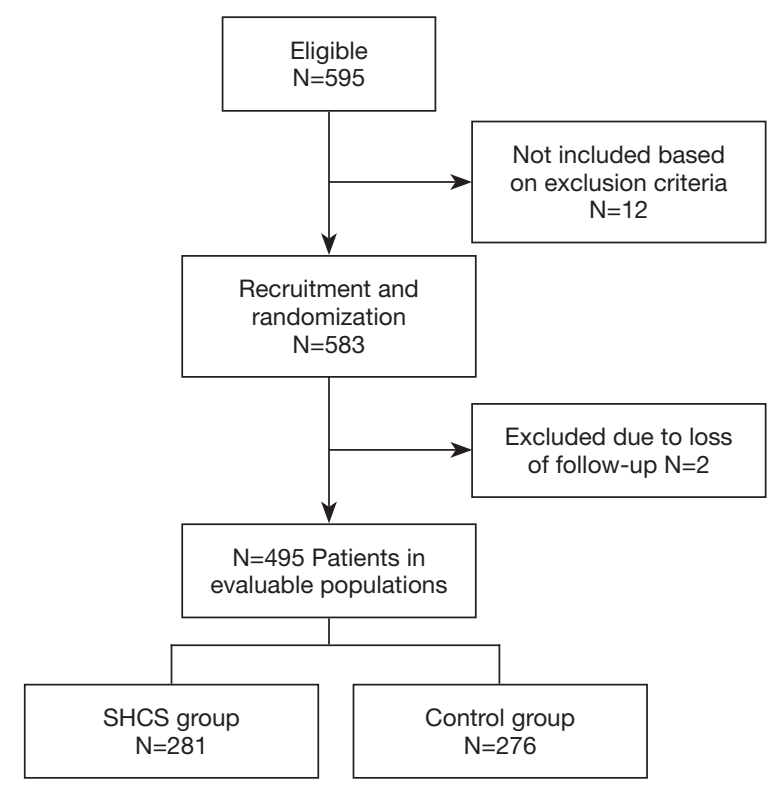

Figure 1 Flow diagram of patient recruitment.

of the patient recruitment process is shown in Figure 1. Inclusion criteria were as follows: (I) diagnosed with primary lung cancer; (II) underwent VATS lobectomy; (III) no chemical thromboprophylaxis received before or after surgery; (IV) at least 18 years of age; and (V) signed written informed consent. Exclusion criteria were: (I) presence of previous VTE and coagulation disorders; (II) uncontrolled cardiovascular disease; or (III) underwent intraoperative conversion to thoracotomy.

\section{Study protocol}

On the day of admission, patients were randomly assigned to the SHCS group or the control group using sealed envelopes containing a slip indicating the allocation, which had been derived from a computer-generated sequence. All of the participants received routine nursing measures and performed preoperatively and postoperative foot and ankle exercises. The participants in the SHCS group, had SHCSs (Figure 2), which were made of self-heating element and cotton, applied to both legs between anesthesia induction and patient positioning. The protocol was approved by the Institutional Review Board (IRB) of Shanghai Pulmonary Hospital (Tongji University) (No. K18-126). The study was conducted in accordance with the Declaration of Helsinki (as revised in 2013). 

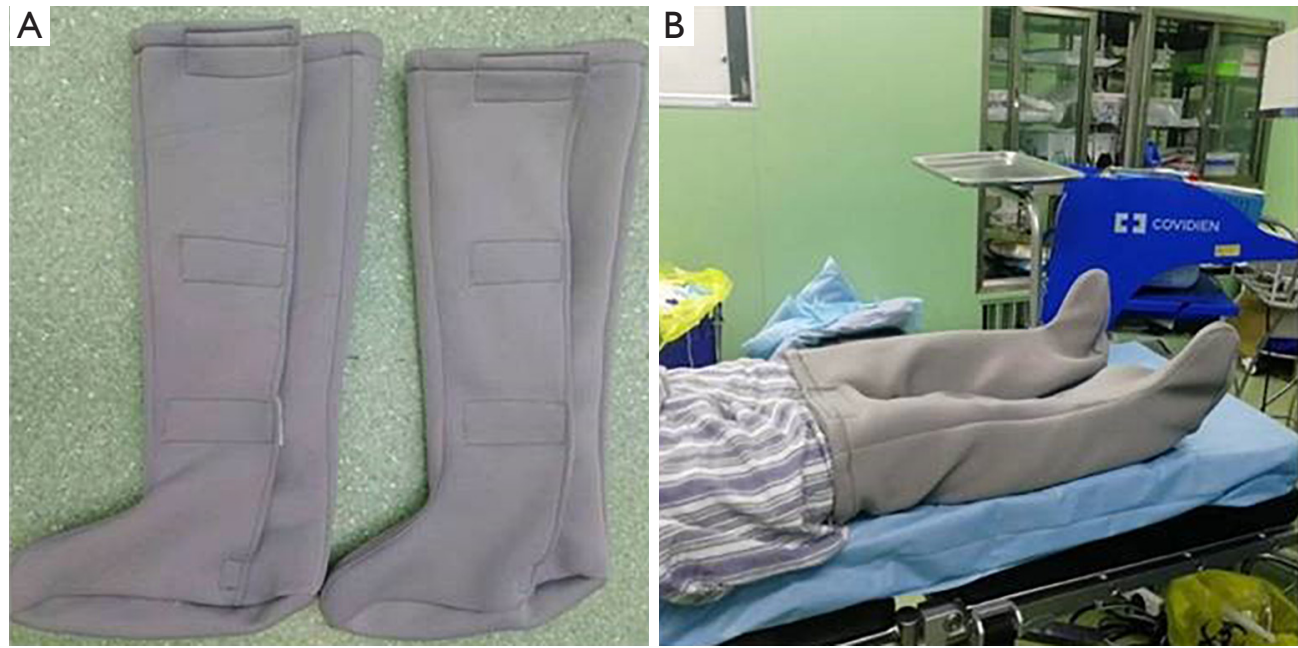

Figure 2 Representative images of self-heating calf sleeves (SHCSs). A pair of SHCSs (A). Administration of the SHCSs to a patient (B).

\section{Outcome measurements}

Postoperatively, the patients underwent close observation for signs and symptoms of DVT. Signs and symptoms flagged as suspicious included redness or swelling in the foot and ankle, pain or tenderness in the leg, and dyspnea. Plasma D-dimer level was determined by Enzyme-linked immunosorbent assay (ELISA). A D-dimer ELISA kit (VIDAS, Biomerieux, France) was used to measure D-dimer concentration according to the manufacturer's instructions. The absorbance was read in an automated immunoassay analyzer (VIDAS, Biomerieux, France) at a wavelength of $450 \mathrm{~mm}$. The D-dimer concentration was obtained by extrapolation from the standard calibration curve obtained by plotting the absorbance against the concentration of the reference standard. The cutoff value was set at $500 \mathrm{ng} / \mathrm{mL}$. Color Doppler ultrasonography was carried out by the same examiner who was blinded to the group status preoperatively and postoperatively to determine the presence or absence of DVT. Blood flow in the lower limbs was detected three times preoperatively and postoperatively with a Doppler ultrasound by a 4-8 MHz linear transducer. The mean of the three measurements was used for analysis. Primary outcomes were any DVT as determined by bilateral ultrasonography on postoperative day 2 .

\section{Statistical analysis}

The sample size was calculated based on the assumption that without prophylaxis, about $16 \%$ of the patients develop DVT (9) and 5\% of patients using SHCS would develop
DVT. This calculation was performed by chi-square test, assuming an $\alpha$ value of 0.05 and a power of $80 \%$.

The patients' baseline characteristics were tested for normal distribution. Continuous variables were expressed as mean \pm standard deviation (SD) and categorical variables were expressed as counts and proportions. Differences in categorical and continuous variables were compared by Student's $t$-test and the $\chi^{2}$ test. All reported $\mathrm{P}$ values were two-tailed. All statistical analyses were performed using SPSS version 22 software (SPSS, Chicago, IL, USA). $\mathrm{P}<0.05$ was considered to represent statistical significance.

\section{Results}

No SHCS-related complications were observed in this study.

\section{Baseline characteristics of the patients}

The baseline characteristics of the included patients are summarized in Table 1. The risk of DVT was individually assessed for each patient based on risk factors, including sex, age, and comorbidities. There were no significant differences in baseline characteristics, operation time, or duration of hospital stay between the SHCS group and the control group.

\section{Incidence of $\mathrm{DVT}$}

Table 2 shows the incidence of DVT in the entire cohort. Of 
Table 1 Baseline characteristics of patients who underwent VATS

\begin{tabular}{lccc}
\hline Variable & SHCS $(\mathrm{n}=281)$ & Control $(\mathrm{n}=276)$ & $\mathrm{P}$ value \\
\hline Gender (male/female) & $135 / 146$ & $133 / 143$ & 0.9726 \\
Age $(\mathrm{y})$ & $64.54 \pm 10.13$ & $64.82 \pm 6.265$ & 0.6956 \\
$\mathrm{BMI}\left(\mathrm{kg} / \mathrm{m}^{2}\right)$ & $23.77 \pm 8.001$ & $23.55 \pm 3.047$ & 0.6640 \\
Smoking & $77(27.4 \%)$ & $80(29.0 \%)$ & 0.6779 \\
Hypertension & $51(18.1 \%)$ & $52(18.8 \%)$ & 0.8336 \\
Diabetes & $18(6.4 \%)$ & $14(5.1 \%)$ & 0.4990 \\
Blood transfusion & $1(0.4 \%)$ & $1(0.4 \%)$ & 0.9795 \\
Operation time (min) & $107.4 \pm 33.70$ & $109.6 \pm 34.99$ & 0.4501 \\
Length of hospital stay (day) & $7.367 \pm 2.636$ & $7.717 \pm 2.521$ & 0.1091 \\
\hline
\end{tabular}

Values are presented as mean \pm SD or number (\%). VATS, video-assisted thoracoscopic surgery; SHCS, self-heating calf sleeve; BMI, body mass index.

Table 2 Incidence of DVT in the SHCS and control group

\begin{tabular}{lcccccc}
\hline Group & Non-DVT & DVT & Total & DVT incidence & OR $(95 \%$ Cl) & P value \\
\hline Control & 234 & 42 & 276 & $15.20 \%$ & 1 (reference) \\
SHCS & 269 & 12 & 281 & $4.27 \%$ & $0.2485(0.1278,0.4834)$
\end{tabular}

DVT, deep vein thrombosis; SHCS, self-heating calf sleeve; OR, odds ratio; Cl, confidence interval.

Table 3 Flow velocity of the femoral vein $(\mathrm{cm} / \mathrm{s}$, mean $\pm \mathrm{SD})$

\begin{tabular}{lccccc}
\hline \multirow{2}{*}{ Group } & \multicolumn{2}{c}{ Pre-operation } & \multicolumn{2}{c}{ Post-operation } \\
\cline { 2 - 5 } & Vmax & Vmin & Vmax & $10.42 \pm 2.64$ \\
SHCS & $21.91 \pm 3.32$ & $7.96 \pm 2.53$ & $23.17 \pm 2.18$ & $6.73 \pm 2.94$ \\
Control & $22.34 \pm 3.18$ & $8.23 \pm 2.64$ & $20.13 \pm 1.93$ & 0.0085 \\
P value & 0.7708 & 0.8180 & 0.0040 & \\
\hline
\end{tabular}

SHCS, self-heating calf sleeve.

the 557 patients included in this study, 54 developed DVT. In the control group, 42 of the 276 patients developed DVT (15.20\%), compared to 12 out of 281 patients $(4.27 \%)$ in the SHCS group. There was a significant difference in the incidence of DVT between the two groups $(\mathrm{P}<0.0001)$.

\section{Lower limb bemodynamics}

As shown in Table 3, the preoperative flow velocity of the femoral vein in the SHCS group was comparable to that in the control group. While the postoperative blood flow in the femoral vein was decreased in the control group, the blood flow in the femoral vein was enhanced by intraoperative administration of SHCSs, which demonstrated the positive impact of the SHCSs on blood flow in the lower extremities.

\section{Plasma D-dimer concentration}

As shown in Figure 3, the preoperative D-dimer concentration in the SHCS group $(168.4 \pm 224.3 \mathrm{ng} / \mathrm{mL})$ was comparable to that in the control group $(221.0 \pm 723.5 \mathrm{ng} / \mathrm{mL})$. On day 2 post-operation, D-dimer concentration was significantly elevated in both groups $(554.9 \pm 927.6 \mathrm{ng} / \mathrm{mL}$ in the SHCS group and $662.4 \pm 972.4 \mathrm{ng} / \mathrm{mL}$ in the control group); however, no significant difference was found. 


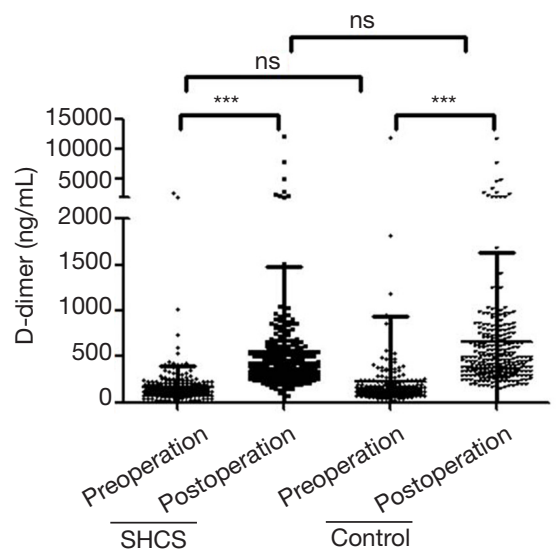

Figure 3 Plasma D-dimer concentration in the self-heating calf sleeves (SHCSs) and the control group. ${ }^{* * *}, \mathrm{P}<0.001$. ns, not significant.

\section{Discussion}

DVT, a common surgical complication, is one of the leading causes of death in cancer patients who undergo surgery. Subsequent complications of DVT, such as postthrombotic syndrome, can profoundly impact a patient's quality of life (11). Even in an era of minimally invasive surgery and advanced perioperative care, the development of DVT still poses a high risk for lung cancer patients who receive surgery (12). Therefore, routine prophylaxis is essential for lung cancer patients who receive surgery.

In this study, we evaluated the efficacy of SHCSs in preventing DVT in lung cancer patients who underwent VATS lobectomy. The incidence of postoperative DVT decreased from $15.20 \%$ in the control group to $4.27 \%$ in the SHCS group without causing any complication such as bleeding. Furthermore, SHCSs significantly improved the venous blood flow, which was shown by hemodynamic assessment. However, there was no statistical difference between the $\mathrm{D}$-dimer levels in the two groups. This was most likely a consequence of the low specificity nature of the test itself, as D-dimer elevation can be caused by several factors, including anesthetic stimulation, tumor, surgical injury, and older age $(13,14)$. In our previous study, we found that IPC could reduce the incidence of DVT to $0.8 \%$ (15), which indicated that SHCSs give a more modest performance in DVT prevention than IPC.

Optimal thromboprophylaxis in patients who undergo major surgery should take into account the risks of DVT and adverse effects, as well as the financial cost and patient compliance. While SHCSs might be less effective than IPC in reducing the risk of DVT, SHCSs have more favorable usability characteristics and are not accompanied by the issues seen with IPC, such as cutaneous complications and noise $(16,17)$, which would make a higher level of patient compliance likely. SHCSs are also cheaper than IPC (the cost of a pair of SHCSs is $¥ 168$, while IPC costs $¥ 45,000$ ). In addition, SHCSs are portable and their application does not require clinical expertise, which makes them an attractive alternative to IPC for use at home.

One major limitation of the present study was that due to the short duration of hospital stay of the patients who received VATS, ultrasonography was only performed on day 2 post-operation. With up to $23 \%$ of postoperative thrombotic events occurring after discharge (18), some DVT cases which occurred in the post-discharge period may have been missed in this study.

In conclusion, our results demonstrate that SHCSs are a satisfactory form of DVT prophylaxis that can improve venous blood flow and thus alleviate venous stasis in the lower extremities. SHCSs offer a simple, inexpensive, and generally complication-free prophylactic method for surgical patients, presenting a viable alternative to IPC in both the clinical and home settings.

\section{Acknowledgments}

The authors thank all the patients who participated in this study and the clinical managers and nurses who provided support.

Funding: This work was supported by funding from Shanghai Pulmonary Hospital (HL201704).

\section{Footnote}

Reporting Checklist: The authors have completed the CONSORT reporting checklist. Available at http://dx.doi. org/10.21037/apm-20-1165

Data Sharing Statement: Available at http://dx.doi. org/10.21037/apm-20-1165

Conflicts of Interest: All authors have completed the ICMJE uniform disclosure form (available at http://dx.doi. org/10.21037/apm-20-1165). The authors have no conflicts of interest to declare.

Ethical Statement: The authors are accountable for all aspects of the work in ensuring that questions related to the accuracy or integrity of any part of the work are 
appropriately investigated and resolved. The protocol was approved by the Institutional Review Board (IRB) of Shanghai Pulmonary Hospital (Tongji University) (No. K18-126). All participants signed the written informed consent. The study was conducted in accordance with the Declaration of Helsinki (as revised in 2013).

Open Access Statement: This is an Open Access article distributed in accordance with the Creative Commons Attribution-NonCommercial-NoDerivs 4.0 International License (CC BY-NC-ND 4.0), which permits the noncommercial replication and distribution of the article with the strict proviso that no changes or edits are made and the original work is properly cited (including links to both the formal publication through the relevant DOI and the license). See: https://creativecommons.org/licenses/by-nc-nd/4.0/.

\section{References}

1. Cosmi B, Palareti G. "Early thrombus removal" in iliacfemoral deep vein thrombosis for prevention of postthrombotic syndrome. Ann Transl Med 2019;7:S343.

2. Trinh VQ, Karakiewicz PI, Sammon J, et al. Venous thromboembolism after major cancer surgery: temporal trends and patterns of care. JAMA Surg 2014;149:43-9.

3. EINSTEIN-PE Investigators, Büller HR, Prins MH, et al. Oral rivaroxaban for the treatment of symptomatic pulmonary embolism. N Engl J Med 2012;366:1287-97.

4. Di Nisio M, van Es N, Buller HR. Deep vein thrombosis and pulmonary embolism. Lancet 2016;388:3060-73.

5. Tagalakis V, Levi D, Agulnik JS, et al. High risk of deep vein thrombosis in patients with non-small cell lung cancer: a cohort study of 493 patients. J Thorac Oncol 2007;2:729-34.

6. Wang X, Song S, Ni R, et al. Effectiveness of intraoperative administration of intermittent pneumatic compression in preventing deep vein thrombosis in lung cancer patients undergoing video-assisted thoracoscopic surgery lobectomy. J Thorac Dis 2019;11:2832-8.

7. Zhang Y, Yang Y, Chen W, et al. Prevalence and associations of VTE in patients with newly diagnosed lung cancer. Chest 2014;146:650-8.

8. Papageorgiou C, Vandreden P, Marret E, et al. Lobectomy and postoperative thromboprophylaxis with enoxaparin improve blood hypercoagulability in patients with localized primary lung adenocarcinoma. Thromb Res 2013;132:584-91.

9. Tian B, Song C, Li H, et al. The significance of perioperative coagulation and fibrinolysis related parameters after lung surgery for predicting venous thromboembolism: a prospective, single center study. J Thorac Dis 2018;10:2223-30.

10. Guanella R, Ducruet T, Johri M, et al. Economic burden and cost determinants of deep vein thrombosis during 2 years following diagnosis: a prospective evaluation. J Thromb Haemost 2011;9:2397-405.

11. Spyropoulos AC, Hurley JS, Ciesla GN, et al. Management of acute proximal deep vein thrombosis: pharmacoeconomic evaluation of outpatient treatment with enoxaparin vs inpatient treatment with unfractionated heparin. Chest 2002;122:108-14.

12. Song C, Shargall Y, Li H, et al. Prevalence of venous thromboembolism after lung surgery in China: a single-centre, prospective cohort study involving patients undergoing lung resections without perioperative venous thromboembolism prophylaxis†. Eur J Cardiothorac Surg 2019;55:455-60.

13. Torbicki A, Perrier A, Konstantinides S, et al. Guidelines on the diagnosis and management of acute pulmonary embolism: the Task Force for the Diagnosis and Management of Acute Pulmonary Embolism of the European Society of Cardiology (ESC). Eur Heart J 2008;29:2276-315.

14. Bruinstroop E, van de Ree MA, Huisman MV. The use of D-dimer in specific clinical conditions: a narrative review. Eur J Intern Med 2009;20:441-6.

15. Wang X, Song S, Ni R, et al. Effectiveness of intraoperative administration of intermittent pneumatic compression in preventing deep vein thrombosis in lung cancer patients undergoing video-assisted thoracoscopic surgery lobectomy. J Thorac Dis 2019;11:2832-8.

16. CLOTS (Clots in Legs Or s'Tockings after Stroke) Trials Collaboration, Dennis M, Sandercock P, et al. Effectiveness of intermittent pneumatic compression in reduction of risk of deep vein thrombosis in patients who have had a stroke (CLOTS 3): a multicentre randomised controlled trial. Lancet 2013;382:516-24.

17. Comerota AJ, Katz ML, White JV. Why does prophylaxis with external pneumatic compression for deep vein thrombosis fail? Am J Surg 1992;164:265-8.

18. Merkow RP, Bilimoria KY, McCarter MD, et al. Post-discharge venous thromboembolism after cancer surgery: extending the case for extended prophylaxis. Ann Surg 2011;254:131-7.

Cite this article as: Chen $\mathrm{S}, \mathrm{Xu}$ W, Song S, Wang X. Efficacy of self-heating calf sleeves for preventing deep vein thrombosis in lung cancer patients who undergo video-assisted thoracoscopic surgery lobectomy. Ann Palliat Med 2020;9(5):26932698. doi: 10.21037/apm-20-1165 